\title{
Einmal MKG-Chirurgie, bitte!
}

\author{
Nach meinem Medizinstudium und der Approbation fiel die Entscheidung: „Ich möchte Fachärztin für \\ MKG-Chirurgie werden." Nun manage ich meinen Alltag zwischen Berufstätigkeit und Zweitstudium.
}

\section{Dr. Lena Müller //}

ist approbierte Ärztin und studiert

Zahnmedizin im 8. Semester. Seit Ausgabe

1/2017 berichtet sie über ihre Erfahrungen mit

Doppelstudium, Karrierewunsch MKG-

Chirurgie und ehrenamtliches Engagement.

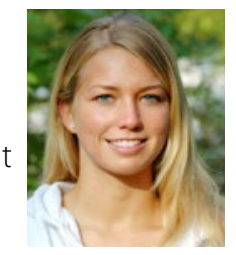

\section{$T_{\text {Ze }}$}<smiles>C1CC1</smiles>

edes Ende ist ein Neuanfang - so auch bei mir: Ich hatte bei zahlreichen Geburten assistiert, Narkosen mit eingeleitet, Zehen amputiert und während meines Medizinstudiums tendenziell eher eine Vorliebe für Unfallchirurgie gehabt - und doch fiel meine Wahl nun auf die Kieferchirurgie. „Willst du wirklich nochmal vier Jahre studieren? In der Praxis macht man ja dann doch das Gleiche wie ein Oralchirurg!"

In der Berufssoziologie spricht man von einer sozialen Vererbungstheorie. Der Beruf, die Branche, die Art und das Niveau der Arbeit der Eltern - all das kann Söhne und Töchter beeinflussen. Und so auch bei mir: Meine Eltern sind beide Zahnärzte in einer Gemeinschaftspraxis. Dieser Umstand hat es mir erlaubt, dass ich bereits als kleines Kind „Behandler" mit Luftpuster und Speichelsauger sein durfte. Ein wichtiges rationales Argument jedoch war die Option, sich als Kieferchirurgin spä- ter relativ gut niederlassen zu können - und die Tatsache, das volle Spektrum der Operationen auch als Frau beherrschen zu können. In der Unfallchirurgie kam ich krafttechnisch häufig an meine körperlichen Grenzen.

Ein erstes Problem meiner neuen Berufswahl war nun die Finanzierung des Zahnmedizinstudiums: Volle Stellen sind aufgrund der Anwesenheitspflicht im Semester nicht möglich und für Zweitstudierende gibt es fast keine Stipendien. Auch eine erneute Studienplatzvergabe durch die ZVS stand mir bevor. Hier gibt es jedoch anders als beim Erststudium ein Punktesystem, welches die Note des Erststudiums, Gründe für die Zweitstudienwahl und weitere individuelle Gegebenheiten berücksichtigt. Ich hatte Glück: eine Platzzusage in meiner Wunschstadt Mainz sowie eine ärztliche Teilzeitstelle in der Diabetologie. Alternativ wäre ich auch bereit gewesen, eine Stelle in einer anderen medizinischen Fachdisziplin anzunehmen - um zu einem späteren Zeitpunkt in die MKG-Chirurgie zu wechseln. Diesen Weg gehen viele angehende Kieferchirurgen, sofern sie nicht direkt eine Stelle in ihrem Wunschfach bekommen.

Trotz aller Hürden und Schichtdienst bin ich mit meiner Entscheidung sehr glücklich und rate meinen Kommilitonen und Kommilitoninnen mit Interesse an MKG-Chirurgie zu dieser Entscheidung - auch wenn der Weg länger ist. Einige meiner Kollegen studieren und arbeiten nicht nur gleichzeitig, sondern haben bereits eine kleine Familie - wenn man möchte, ist also durchaus alles gut zu schaffen!

\section{Impressum}

Verlag: Springer Medizin Verlag GmbH, Heidelberger Platz 3, 14197 Berlin (Betriebsstätte Heidelberg: Springer Medizin Verlag GmbH, Tiergartenstraße 17, 69121 Heidelberg; Tel. +49 6221/487-0), SpringerZahnmedizin.de Geschäftsführer: Joachim Krieger, Fabian Kaufmann Director Apotheke und Zahnmedizin: Marion Bornemann (V. i. S. d. P.)

Redaktionsleiterin Zahnmedizin: Susanne Redeker Redaktionsleitung: Dr. Swanett Koops

(Tel.-8217, swanett.koops@@springernature.com) Redaktionelle Mitarbeit: Katharina Schneider

(Tel. -8488, katharina.schneider@springernature.com Layout: Arnulf Illing (Tel. -8952, arnulf.illing@@springernature.com) Anzeigenleitung: Marita Säuberlich (Tel. -8309, Fax-68309, marita.saeuberlich@springer.com), SpringerMedizin.de/mediadaten Corporate Publishing Zahnmedizin:

Anja Weisse (Tel. -8739, Fax-68739, anja.weisse@springer.com)

Druck: Vogel Druck und Medienservice GmbH, Leibnizstraße 5, 97204 Höchberg

Erscheinungsweise: 4 Ausgaben pro Jahr

Papierausgabe: ISSN 1869-5744, gedruckt auf säurefreiem Papier

Eigentümer \& Copyright: @ S Springer Medizin Verlag GmbH 2017.

Springer Medizin Verlag GmbH ist Teil von Springer Nature.

Die Zeitschrift sowie alle in ihr enthaltenen einzelnen Beiträge und Abbildungen sind urheberrechtlich geschützt. Jede Verwertung, die nicht ausdrücklich vom Urheberrechtsgesetz zugelassen ist, bedarf der vorherigen schriftlichen Zustimmung des Verlags. Das gilt insbesondere für Vervielfältigungen, Bearbeitungen, Übersetzungen, Mikroverfilmungen und die Einspeicherung und Verarbeitung in elektronischen Systemen.
Preis für persönliche Abonnenten inkl. Online-Basis-Lizenz 2017:

(4 Hefte) EUR 60,- (unverb. Preisempfehlung inkl. gesetzlicher MwSt.)

Einzelheftpreis 2017: EUR 24,- (unverb. Preisempfehlung inkl. gesetzlicher MwSt.). Für Studierende und für Ärzte in der Facharztausbildung ermäßigt sich 2017 der Bezugspreis auf jährlich EUR 36,- (unverb. Preisempfehlung inkl. gesetzlicher MwSt., Nachweis über Studium/Ausbildung erforderlich). Institutspreis inkl. Online-Basis-Lizenz 2017: (4 Hefte) EUR 208,83 (unverb. Preisempfehlung inkl. gesetzlicher MwSt.). Der Bezugspreis ist im Voraus zu zahlen. Das Abonnement kann bis 30 Tage vor Ende des Bezugszeitraums gekündigt werden.

Allgemeiner Hinweis zur gesetzlichen Mehrwertsteuer: Gedruckte Zeitschriften unterliegen grundsätzlich dem ermäßigten Steuersatz von 7\%, digitale Produkte (wie z.B. die Online-Version einer Zeitschrift) hingegen dem allgemeinen Steuersatz von 19\%. Die detaillierte Aufteilung der einzelnen Mehrwertsteuer-Beträge entnehmen Sie bitte Ihrer Rechnung. Bestellungen oder Rückfragen nimmt jede Buchhandlung oder der Verlag entgegen. Springer Customer Service Center GmbH, Tiergartenstr. 15-17, 69121 Heidelberg Tel. +49 6221/345-4301 (Mo.-Fr. 8.00 Uhr bis 18.00 Uhr), Fax +496221/345-4229, customerservice@springer.com

Autoren können unter bestimmten Voraussetzungen an der Ausschüttung der Bibliotheks- und Fotokopietantiemen teilnehmen. Einzelheiten bei VG WORT, Abt. Wissenschaft, Goethestr. 49, 80336 München.

Angaben über Dosierungsanweisungen und Applikationsformen sind anhand anderer Literaturstellen oder der Packungsbeilage auf ihre Richtigkeit zu überprüfen. Der Verlag übernimmt keine Gewähr.

Der IVW (Informationsgemeischaft zur Feststellung der Verbreitung von Werbeträgern) angeschlossen. 